\title{
Runaway migration in a multiple-protoplanet system
}

\author{
Hui Zhang and Ji-Lin Zhou \\ Department of Astronomy,Nanjing University,Nanjing 210093,China
}

\begin{abstract}
We investigate the migration of two giant planets embedded in a proto-stellar disk. The inner planet(initially located at $R_{10}=1$ ) is of 1 Jupiter mass and the outer one $\left(R_{20}=1.5\right.$ ) is of 1 Saturn mass. We find that due to the existence of the inner massive planet, the outer planet can not open a clear gap. Instead of an inward migration and being captured by the mean motion resonance of the inner planet, the outer planet undergoes an outward runaway migration. We conclude that this runaway migration is caused by the co-rotation torque in the co-orbital region of the outer planet and sustained by the wave(flow) driven by the inner massive planet.
\end{abstract}

Keywords. Exoplanet, multiple planets, migration, runaway migration

\section{Introduction}

Migration of planets is one of the important processes that affect the final architecture of a planet system. Important migrations such as Type I, Type II and Type III migrations may change substantially the configuration of multiple-planet systems(See Masset 2008 for a review). A protoplanet normally undergoes Type I or Type II migration depending on its mass. These two kinds of migration had been well studied both analytically and numerically during the past years. However, the cause as well as the consequence of Type III migration remains unclear and needs further investigations especially in a multipleplanet system. Linear analysis suggests that the interaction between an embedded planet and the disk leads to a negative torque on the planet, which is called differential Lindblad torque(Goldreich \& Tremaine 1980, Ward 1986). So it is believed that low mass protoplanets will undergo rapid inward migration(Type I) and be trapped in the mean motion resonances of the giant planet in a multiple-planet system(Kley 2000, Cresswell \& Nelson 2006). The first numerical simulation of Jupiter and Saturn embedded in a gas disk has been done by Masset and Snellgrove(2001). They found the Saturn was captured into the 2:3 mean motion resonance with Jupiter. And then the two planets reversed their migration outward in parallel. Morbidelli and Crida (2007) extend their work by exploring a wider set of initial conditions and disk parameters. They found the two planets will eventually end up locked in a MMR. The situation maybe different if planets embedded in a disk where the disk gas is still massive. In such a situation, the co-rotation torque on the protoplanets may arise and play a significant role on the orbital evolution of planets(Masset \& Papaloizou 2003).

In this report, we study the onset of type III migrations in a two-planet system. The inner planet is set to have a mass of 1 Jupiter mass and the outer one is of 1 Saturn mass. The planets are embedded in a massive disk with totally more than 20 Jupiter masses distributed in the disk uniformly. Numerical simulation indicated that the outer planet can not open a clear gap due to the presence of inner planet perturbation, and a rapid runaway outward migration occurs on the outer planet. By comparing with the single-planet case, we conclude that this outward runaway migration is probably caused 
by the existence of inner planet which performs strong perturbation to the outer planet's co-orbital region and results in a strong co-orbital torque on the planet. We present our numerical methods in section 2 and our results in section 3 . In section 4 we summary and discuss the implication of our results.

\section{Physical and Numerical Model}

\subsection{Physical model}

We construct a 2D numerical hydrodynamic scheme to solve the continuity and momentum equations, neglecting the effect of any explicit viscosity. We solve the governing equations in the Cartesian coordinate.

The vertically averaged continuity equation for the disk gas is given by

$$
\frac{\partial \sigma}{\partial t}+\frac{\partial\left(\sigma u_{x}\right)}{\partial x}+\frac{\partial\left(\sigma u_{y}\right)}{\partial y}=0
$$

The equations of motion in the Cartesian coordinates are,

$$
\begin{gathered}
\frac{\partial\left(\sigma u_{x}\right)}{\partial t}+\frac{\partial\left(\sigma u_{x}^{2}\right)}{\partial x}+\frac{\partial\left(\sigma u_{x} u_{y}\right)}{\partial y}=-\frac{\partial P}{\partial x}-\sigma \frac{\partial \Phi}{\partial x} \\
\frac{\partial\left(\sigma u_{y}\right)}{\partial t}+\frac{\partial\left(\sigma u_{x} u_{y}\right)}{\partial x}+\frac{\partial\left(\sigma u_{y}^{2}\right)}{\partial y}=-\frac{\partial P}{\partial y}-\sigma \frac{\partial \Phi}{\partial y}
\end{gathered}
$$

where $\mathrm{P}$ is pressure and $\Phi$ is the gravity potential of the star-planets-disk system, which includes the softened potential of central star $\left(\Phi_{s}\right)$, softened potential of the planets $\left(\Phi_{p i}\right)$, potential of the disk itself $\left(\Phi_{d}\right)$ and indirect potential $\left(\Phi_{i}\right)$ due to the acceleration of origin by the planets and the disk. $\sigma$ is the vertical integrated surface density.

We assume the disk gas has an isothermal equation of state. The sound speed is $c_{s}=(H / r) v_{k}$ where we set $H / r=0.04$ and $v_{k}=1($ Keplerian velocity at $r=1)$. We don't add any explicit viscosity in the simulation. There is however some numerical viscosity associated with our computational scheme and grid effects. To focus on the migration and reduce variables, we don't allow the planet to accrete gas from the disk.

\subsection{Computational Units}

For numerical convenience we set gravitational constant $G=1$, solar mass $M_{\odot}=1$ and the radius of inner planet's initial orbit $R_{10}=1$, where $R_{10}=5.2 \mathrm{AU}$. The unit of time is $1 / 2 \pi$ of the inner planet's initial orbit period $P_{10}$. Total mass within the disk are about 20 Jupiter masses, which corresponds to a uniform surface density $\sigma=0.0006$.

\subsection{Numerical Method}

The Antares code we have developed is adopted in the calculations. It is a 2-D Godunov code based on the exact Riemann solution for isothermal or polytropic gas, featured with non-reflecting boundary conditions. At the boundaries we adopt non-reflecting (absorbing) boundary condition to make sure there is no wave reflect from the boundaries. To avoid initial impact of the system we adopt a 'quiet start' initial condition. For the orbit of planets we use RK78 to integrate it. For more details of the physical and numerical methods please see Zhang et al. 2008.

\section{Numerical Results}

We performed a hydrodynamic simulation to investigate the orbit evolution of two giant planets embedded in a massive disk. The computational domain is from -3 to 3 in 

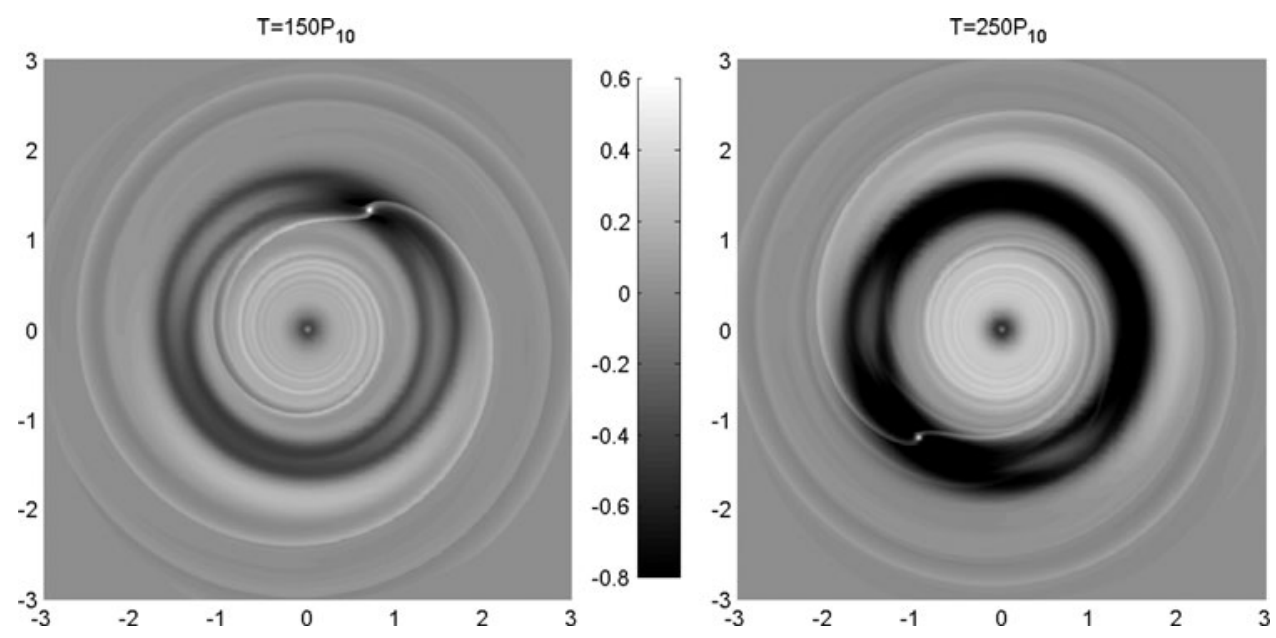

Figure 1. Density evolution of the disk contains only one planet. Without the inner massive planet, the outer planet may open a clear gap when it is of 1 Saturn mass.

$x$ direction and from -3 to 3 in $y$ direction. Total resolution is $N_{x} \times N_{y}=1024 \times 1024$. At beginning the disk is uniform and have 20 Jupiter masses. The initial locations of two planets are $R_{10}=1$ (Jupiter mass) and $R_{20}=1.5$ (Saturn mass). We hold their orbits and increase their mass gently from $1 \times 10^{-7}$ to their final mass. After $200 P_{10}$ which is the orbit period of the inner planet, two planets had grown up and we release them at the same time. In first case, we neglect the inner planet by setting its mass equal to 0 .

As we can see in Fig.1, the (outer)planet opens a gap and a regular, symmetric horseshoe zone appears within its co-orbital region. In second case,we turn on the effects of inner planet. During the first $200 P_{10}$, the inner planet had already form a gap but the outer planet do not clear any gap. Since we set $H / r=0.04$ in our simulations, it will open a clear gap when the embedded planet is above tens of Earth masses(as in the first case). However, the existence of the inner massive planet prevents the outer planet from opening a clear gap. From Fig.2 we can see the inner planet had already open a gap while the outer planet's co-orbital region is still replete of gas.

Further more the waves excited by the two giant planets perturb each other's co-orbital region significantly. During a short period after release, outer planet undergo inward Type I migration and soon be perturbed by the inner planet. Gas flows in and out from its co-orbital region rapidly and form a large vortensity gradient, then this vortensity gradient generates great co-rotation torque on the planet. As a result, the migration of outer planet become unstable. We see a outward runaway migration occurs soon after the release. We also note that during a short period the outer planet is captured into the 5:3 MMR of the inner planet. But as soon as the the outer planet's migration became unstable this MMR is broken up.In the first case when the inner planet is absent, the outer planet is embedded in the gap and undergoes Type II migration which is smooth and slow. See Fig.3-a. 

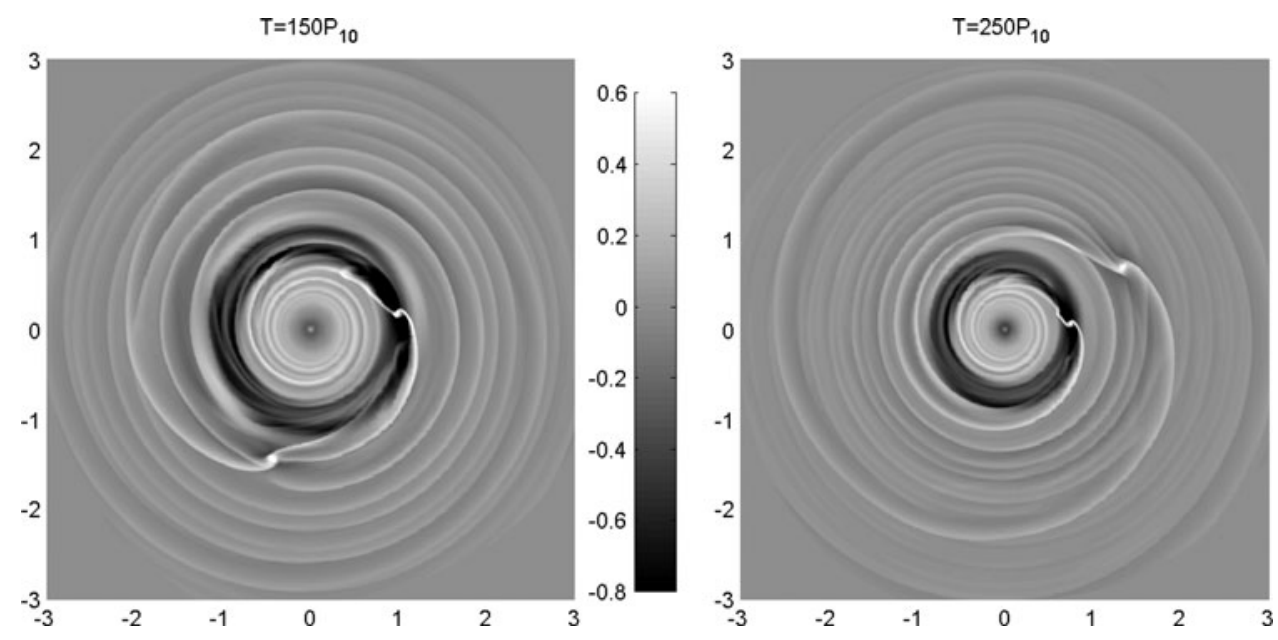

Figure 2. Density evolution of the disk contains two planets. The interaction between the two planets significantly perturbs each planet's co-orbital region, and prevent the outer less massive one from clearing gap.

\section{Conclusion and Discussion}

From the comparison, we can conclude that the runaway migration is triggered by the perturbation comes from the inner planet. Masset \& Papaloizou (2003) have shown that a protoplanet embedded in a massive disk may undergo runaway migration when the co-orbital mass deficit $\delta m$ is comparable with the planet mass $M_{p}$. They analyzed co-rotation torque and gave a relation between the migration rate and co-orbital mass deficit. In a Keplerian case it reads:

$$
\frac{1}{2} a \Omega_{p}\left(M_{p}-\delta m\right) \dot{a}=\Delta \Gamma_{L R}-\frac{\pi a^{2} \delta m}{3 x_{s}} \ddot{a},
$$

where $a$ is the semi-major axis of the planet orbit, $\Omega_{p}$ is the angular velocity of the planet, $\Delta \Gamma_{L R}$ is the differential Lindblad torque and $x_{s}$ is the half-width of planet horseshoe zone. The co-orbital mass deficit $\left(\delta m=M_{H S 0}-M_{H S}\right)$ can be roughly understood as the mass variation within the planet horseshoe zone, where $M_{H S 0}$ is the initial mass within planet horseshoe zone and $M_{H S}$ is the mass within planet horseshoe zone which changes with time. When $\delta m \geqslant M_{p}$, above equation indicates that small perturbation to semi-major axis $(a)$ leads to its exponentially growing on a timescale of few tens of orbital periods. And in specific initial condition $(a$ and $\dot{a})$ outward runaway migration may occur naturally.

A strong perturbation to planet orbit makes gas flush into or out of its co-orbital region and therefor leads to a large $\delta m$. Then the planet will undergo runaway migration triggered by the co-rotation torque. Once the rapid migration occurs, it can self-sustained because the gas will keep flooding in its co-orbital region as it travels a large radial shift within very short time(Zhang et al. 2008).

In our simulations, the existence of the inner giant planet gives strong perturbations to the gas traveling within the outer planet horseshoe region, which leads to a large $\delta m$. Fig.3-b shows the evolution of $M_{H S}$ of each planet. Clearly, the rapid variation of $M_{H S}$ greatly affects the planet migration. As soon as $\delta m$ becomes larger than the 

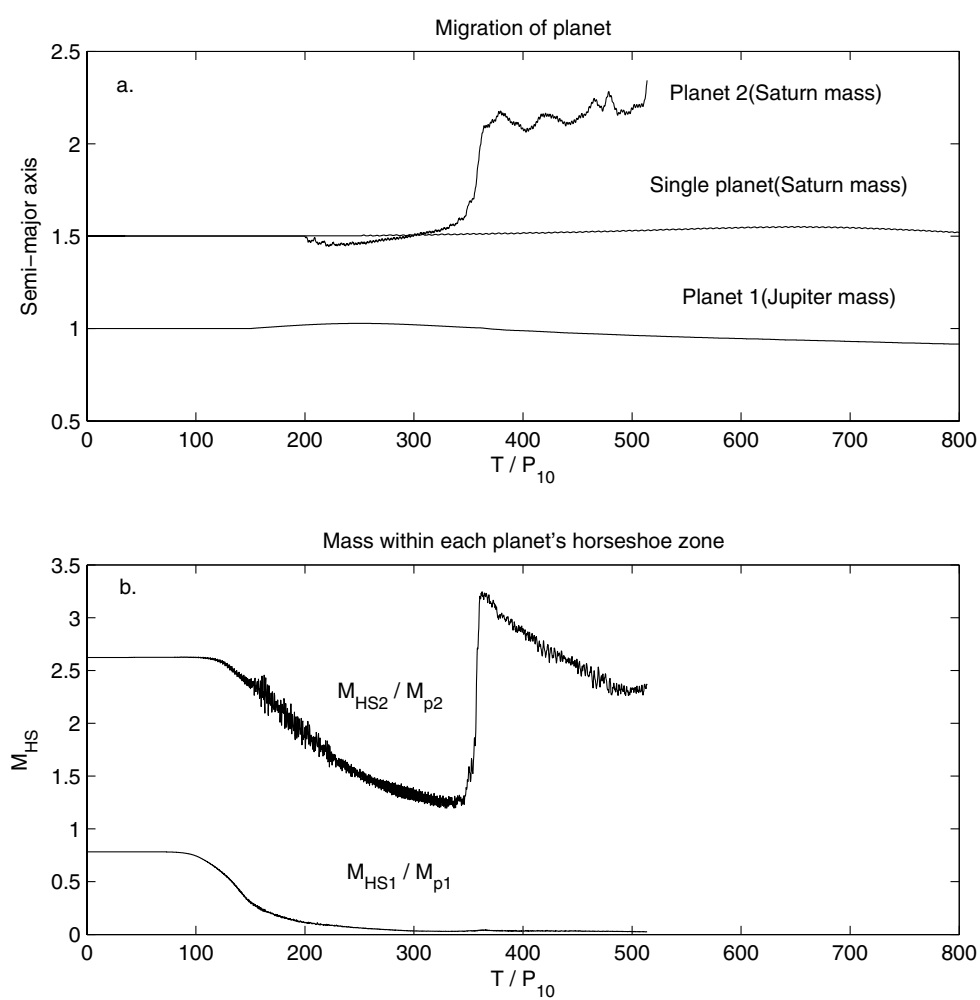

Figure 3. (a).Migration curves of multiple planets system. Two cases are shown in this figure. The first case contains only single Saturn mass planet. The second case contains two planets, one is of a Jupiter mass while the other is of a Saturn mass. (b).Evolution of $M_{H S}$ in second case. Curves are normalized by each planet's mass. Clearly the rapid change of $M_{H S}$ significantly affects the migration of planet 2 . At around $T=350 P_{10}$, a large $\delta m$ appears and leads to the runaway migration of planet 2 .

outer planet $\left(M_{p 2}=3 \times 10^{-4}\right)$, a runaway migration occurs. Within a few orbits after $T=350 P_{10}, M_{H S}$ increases sharply and $\delta m \sim 2 M_{p 2}$. When the planet 2 is far away from planet 1 , this runaway migration can be halted since the perturbation has damped. But its orbit is still unstable. The properties of this kind of runaway migration substantially depends on the initial condition of the system, which merits further studies.

\section{Acknowledgement}

We thank Prof. Lin, D.N.C and Dr. F.S. Masset for very useful discussions. The work is in part supported by a grant from National Basic Research Program of China (2007CB4800), Natural Science Foundation of China (10778603,10403004).

\section{References}

Cresswell, P.\& Nelson, R. P. 2006, A\& A, 450, 833

Goldreich, P. \& Tremaine, S. 1980, ApJ, 241, 441

Kley, W. 2000,MNRAS, 313, 47 
Masset, F. S. \& Snellgrove, M. 2001, MNRAS, 320, L55-L59

Masset, F. S. \& Papaloizou, J. C. B. 2003, ApJ, 588, 494

Masset, F. S. 2008, in IAU Symposium No.249. Exoplanets: Detection, Formation and Dynamics, Y.-S. Sun, S. Ferraz-Mello \& J.-L. Zhou, eds.( Suzhou, China), p. 331

Morbidelli, A. \& Crida, A. 2007, Icarus, 191, 158

Ward, W. R. 1986, ICARUS, 67, 164

Zhang, H., Yuan,C., Lin, D. N. C. \& Yen, D. C. C. 2008, ApJ, (acceptted), Arxivastroph:0709.0338. 